\title{
Localization of a random copolymer at an interface: an exact enumeration study
}

\author{
E W James ${ }^{1}$, C E Soteros ${ }^{1,2}$ and S G Whittington ${ }^{1}$ \\ ${ }^{1}$ Department of Chemistry, University of Toronto, Toronto, M5S 3H6, Canada \\ ${ }^{2}$ Mathematical Sciences Group, University of Saskatchewan, Saskatoon, S7N 5E6, Canada \\ E-mail: ejames@chem.utoronto.ca,soteros@math.usask.ca and swhittin@chem.utoronto.ca
}

Received 13 June 2003

Published 5 November 2003

Online at stacks.iop.org/JPhysA/36/11575

\begin{abstract}
We consider a self-avoiding walk on the simple cubic lattice, as a model of localization of a random copolymer at an interface between two immiscible liquids. The vertices of the walk are coloured $A$ or $B$ randomly and independently. The two liquid phases are represented by the two half-spaces $z>0$ and $z<0$, and the plane $z=0$ corresponds to the interface between the two liquids. The energy depends on the numbers of $A$-vertices with positive $z$-coordinate and $B$-vertices with negative $z$-coordinate. In addition there is a vertex-interface interaction, irrespective of the colour of the vertex. We use exact enumeration and series analysis techniques to investigate the form of the phase diagram and how it changes as the magnitude of the vertex-interface interaction changes.
\end{abstract}

PACS number: $05.40 . \mathrm{Fb}$

\section{Introduction}

Random copolymers are an interesting example of a system with quenched randomness (Brout 1959) since the sequence of comonomers is determined by a random process but is then fixed. Different copolymer molecules will have different monomer sequences so an average over the possible sequences is necessary in order to determine thermodynamic and other properties.

In this paper, we are interested in the localization of a random copolymer at the interface between two immiscible liquids, which we call the $\alpha$-phase and the $\beta$-phase. A typical situation would be two liquids such as oil and water and a copolymer with two types of monomers which are respectively hydrophilic and lyophilic. At high temperatures the polymer might delocalize into the energetically most favourable phase while at low temperatures it will localize at and near the interface. We shall represent the polymer molecules by self-avoiding walks on the simple cubic lattice with vertices of the walk coloured $A$ or $B$ (randomly and independently) to represent the two types of comonomers. For most of this paper we shall take the probability 
$p$ that a vertex is coloured $A$ to be $1 / 2$. There is an energy term for monomers of type $A$ in the $\alpha$-phase and for monomers of type $B$ in the $\beta$-phase. In addition there is an interaction of both types of monomer with the interfacial plane separating the two liquid phases.

Localization of a random copolymer at an interface has been investigated by Sinai and Spohn (1966), Bolthausen and den Hollander (1997), Maritan et al (1999), Biskup and den Hollander (1999), Martin et al (2000) and Madras and Whittington (2003). The model considered here is an extension of that introduced by Martin et al (2000) and has also been investigated by Madras and Whittington (2003). In particular, Martin et al derived several qualitative features of the phase diagram in the absence of a vertex-interface interaction, and estimated the locations of the phase boundaries for this case, using numerical approaches. Madras and Whittington derived additional qualitative results about the shapes of the phase boundaries and extended these results to the case with a non-zero vertex-interface interaction. The primary aim of this paper is to determine, using exact enumeration and series analysis techniques, the form of the phase diagram and how it depends on the vertex-interface interaction.

We consider the simple cubic lattice $\mathbb{Z}^{3}$ and write $(x, y, z)$ for the coordinates of a vertex of the lattice. The $\alpha$-phase is represented by the half-space $z>0$, the $\beta$-phase by the halfspace $z<0$ and the interface by the plane $z=0$. We consider $n$-edge self-avoiding walks with vertices numbered $0,1,2, \ldots, n$ with the zeroth vertex fixed at the origin. Since this vertex is fixed its colour is irrelevant. The vertices $i=1,2, \ldots, n$ are independently coloured $A$ with probability $p$ and $B$ with probability $1-p$. We write $\chi_{i}$ for the colouring $(A$ or $B)$ of the $i$ th vertex and $\chi$ as a shorthand for $\left\{\chi_{1}, \chi_{2}, \ldots, \chi_{n}\right\}$. Suppose that $c_{n}\left(v_{A}, v_{B}, w \mid \chi\right)$ is the number of $n$-edge self-avoiding walks, starting at the origin, with colouring $\chi$, having $v_{A}$ vertices coloured $A$ with positive $z$-coordinate, $v_{B}$ vertices coloured $B$ with negative $z$-coordinate and $w$ vertices (of either colour) with $z$-coordinate equal to zero. We define the partition function

$$
Z_{n}(\alpha, \beta, \gamma \mid \chi)=\sum_{v_{A}, v_{B}, w} c_{n}\left(v_{A}, v_{B}, w \mid \chi\right) \mathrm{e}^{\alpha v_{A}+\beta v_{B}+\gamma w}
$$

and the corresponding free energy

$$
\kappa_{n}(\alpha, \beta, \gamma \mid \chi)=n^{-1} \log Z_{n}(\alpha, \beta, \gamma \mid \chi) .
$$

We now give a brief summary of what is known rigorously. The proofs can be found in Martin et al (2000) and Madras and Whittington (2003). Suppose that $c_{n}$ is the number of self-avoiding walks on the simple cubic lattice, starting at a specified point (say the origin). Then the connective constant (Hammersley 1957) of the lattice is

$$
\kappa_{3}=\lim _{n \rightarrow \infty} n^{-1} \log c_{n}
$$

The quenched average free energy is the average of $\kappa_{n}(\alpha, \beta, \gamma \mid \chi)$ over all colourings $\chi$ and it is known that its limit

$$
\bar{\kappa}(\alpha, \beta, \gamma)=\lim _{n \rightarrow \infty}\left\langle\kappa_{n}(\alpha, \beta, \gamma \mid \chi)\right\rangle
$$

exists, where the angular brackets denote an average over colourings. It is also known that $\bar{\kappa}(\alpha, \beta, \gamma)=\kappa_{3}+\alpha p$ when $\alpha \geqslant 0, \beta \leqslant 0, \gamma \leqslant 0$ and $\bar{\kappa}(\alpha, \beta, \gamma)=\kappa_{3}+\beta(1-p)$ when $\alpha \leqslant 0, \beta \geqslant 0, \gamma \leqslant 0$. The first case corresponds to delocalization into the $\alpha$-phase $(z>0)$ and the region of $(\alpha, \beta, \gamma)$-space for which this equality holds is denoted $D_{\alpha}$. The second case corresponds to delocalization into the $\beta$-phase $(z<0)$ and the corresponding region of $(\alpha, \beta, \gamma)$-space is denoted $D_{\beta}$. Since these two expressions are lower bounds on $\bar{\kappa}$ for all $\alpha, \beta, \gamma$, we say that the walk is delocalized if

$$
\bar{\kappa}(\alpha, \beta, \gamma)=\kappa_{3}+\max \{\alpha p, \beta(1-p)\}
$$


and localized if

$$
\bar{\kappa}(\alpha, \beta, \gamma)>\kappa_{3}+\max \{\alpha p, \beta(1-p)\} .
$$

To simplify the presentation we now specialize to the case $p=1 / 2$. For $\gamma \leqslant 0$ there is a phase boundary $\beta=\beta_{c}(\alpha, \gamma)$ in the first octant of the $(\alpha, \beta)$-plane, where $\bar{\kappa}(\alpha, \beta, \gamma)$ is singular. See figure 1 of Madras and Whittington (2003) for a schematic picture of the phase boundary for $\gamma \leqslant 0$. This phase boundary passes through the origin, has a horizontal asymptote as $\alpha \rightarrow \infty$ and is a concave non-decreasing function of $\alpha$. There is a symmetryrelated phase boundary in the second octant (obtained by reflection in the line $\beta=\alpha$ ) and these two phase boundaries meet only at the origin. There are similar phase boundaries in the fifth and sixth octants and the boundary in the fifth (sixth) octant has a horizontal (vertical) asymptote as $\alpha \rightarrow-\infty(\beta \rightarrow-\infty)$. If we consider $\gamma \geqslant 0$ then there exists $\gamma_{1} \geqslant 0$ such that for all $\gamma>\gamma_{1}$ the two phase boundaries do not have any common points (indeed, for each $\alpha$, the point $(\alpha, \alpha, \gamma)$ is in the interior of the localized region). See figure 2 of Madras and Whittington (2003) for a schematic picture of the phase boundary for $\gamma>\gamma_{1}$. It is known that $\gamma_{1} \leqslant 2 \kappa_{3}-\kappa_{2}-\sinh ^{-1} \cosh \kappa_{3}$ where $\kappa_{2}$ is the connective constant of the square lattice (Hammersley et al 1982, Madras and Whittington 2003, equation 2.5). Moreover, there exists $\gamma_{2}$ such that for all $\gamma>\gamma_{2}$ the system is localized at all points in the third quadrant of the $(\alpha, \beta)$-plane, and $\gamma_{2} \leqslant \kappa_{3}-\kappa_{2}$ (Madras and Whittington 2003, theorem 5(v)). Finally there exists $\gamma_{3}$ such that for all $\gamma>\gamma_{3}$ the whole $(\alpha, \beta)$-plane is in the localized phase and $\log 2 \leqslant \gamma_{3} \leqslant 4 \kappa_{3}$ (Madras and Whittington 2003, theorem 11).

\section{The form of the phase diagram}

We have used exact enumeration data (originally derived in Martin et al (2000)) and ratio analysis methods (Gaunt and Guttmann 1974) to estimate the values of $\bar{\kappa}(\alpha, \beta, \gamma)$ and hence to investigate the form of the phase diagram in the $(\alpha, \beta)$-plane for various values of $\gamma$. If we start at a point in the fourth quadrant where the system is delocalized into the $\alpha$-phase, then we know that $\bar{\kappa}(\alpha, \beta, \gamma)=\kappa_{3}+\alpha / 2$ independent of $\beta$. Fixing $\alpha$ and $\gamma$ we can increase $\beta$ and attempt to locate the last value $\beta=\beta_{c}(\alpha, \gamma)$ for which $\bar{\kappa}=\kappa_{3}+\alpha / 2$; hence for $\alpha \geqslant \beta \geqslant \beta_{c}(\alpha, \gamma)$ the system is localized. Following this strategy we find the approximate values of $\beta_{c}(\alpha, \gamma)$ for a variety of values of $\alpha$ at fixed $\gamma$. In a similar way one can decrease $\alpha$ at fixed $\beta$ and $\gamma$ to cross the phase boundary from a different direction.

A standard assumption for self-avoiding walk models would be that $\left\langle\kappa_{n}(\alpha, \beta, \gamma \mid \chi)\right\rangle$ approaches $\bar{\kappa}$ as $n \rightarrow \infty$ with a correction term of order $(\log n) / n$ (see, for example, Vanderzande (1998)). If we define

$$
Q_{n}=\mathrm{e}^{n\left\langle\kappa_{n}(\alpha, \beta, \gamma \mid x)\right\rangle}
$$

then a simple assumption for the behaviour of $Q_{n}$, for large $n$, is

$$
Q_{n}=A \mathrm{e}^{\bar{\kappa} n} n^{\theta-1}\left(1+B n^{-1}+o\left(n^{-1}\right)\right) \text {. }
$$

Defining $R_{n}=\sqrt{Q_{n} / Q_{n-2}}$ we have, from equation (2.2),

$$
R_{n}=\mathrm{e}^{\bar{\kappa}}\left(1+\frac{\theta-1}{n}+o\left(n^{-1}\right)\right)
$$

and $\bar{\kappa}$ can be estimated by plotting $R_{n}$ against $1 / n$ and extrapolating to large $n$. Nonlinear behaviour can arise from the presence of confluent singularities, i.e. from terms in (2.2) of the form $n^{-\Delta}$ with $\Delta<1$. When the system is in the localized phase the exponent $\theta$ should be characteristic of two-dimensional behaviour so we expect that $\theta=43 / 32$. In the delocalized phases, the walk should be essentially in a half-space so we expect that $\theta$ is about 0.68 


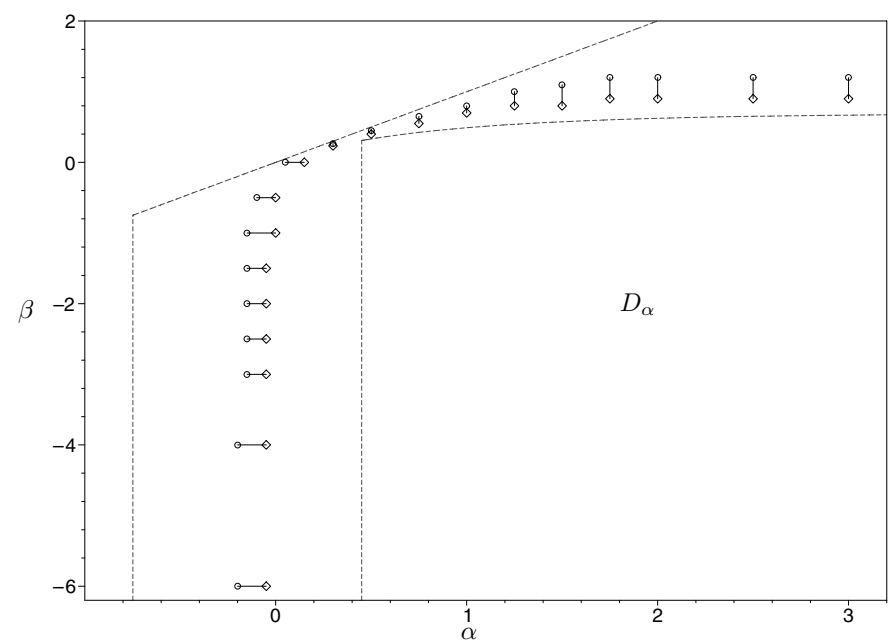

Figure 1. The phase diagram in the $(\alpha, \beta)$-plane for $\gamma=0.2$ and $p=1 / 2$. The dashed lines are bounds on the location of the phase boundary. Solid lines indicate regions that the phase boundary, $\beta_{c}(\alpha, \gamma)$, is estimated to intersect. A $\diamond$ is used to mark a point which is estimated to be in $D_{\alpha}$ and a $\circ$ marks a point estimated to be in the localized phase. Only the estimated phase boundary between the localized region and the delocalized region $D_{\alpha}$ is shown; the other phase boundary can be obtained by reflection in the line $\beta=\alpha$.

(see, e.g. De'Bell and Lookman 1993). We can make use of this information to construct biased estimates of $\bar{\kappa}$. If, for a triple $(\alpha, \beta, \gamma)$, we know $\theta$ exactly then

$$
Y_{n}(\theta)=\frac{n R_{n}}{n+\theta-1}=\mathrm{e}^{\bar{\kappa}}\left(1+o\left(n^{-1}\right)\right)
$$

so that $Y_{n}(\theta)$ approaches $\mathrm{e}^{\bar{\kappa}}$ with zero slope when extrapolated against $n^{-1}$. (Again, deviations from zero slope can be attributed to confluent terms.) For $\alpha, \beta, \gamma$ values well inside either the localized or delocalized regions, this behaviour (with the appropriate value of $\theta$ ) can confirm the nature of the phase. Close to the phase boundary, however, the situation is less clear but these additional functions still give useful information and can help in estimating the value of $\bar{\kappa}$.

We have investigated only one phase boundary since the other is derivable by reflection in the line $\beta=\alpha$. In figure 1 we show our estimate of the location of the phase boundary for $\gamma=0.2$ based on exact values of $Q_{n}$ for $n \leqslant 20$. The vertical dashed line in the third quadrant and the unit slope line through the origin are bounds on the location of the phase boundary, obtained from the results of Martin et al (2000) and Madras and Whittington (2003). More specifically, the vertical dashed line in the third quadrant is based on

$$
\alpha=2\left(\gamma-\kappa_{3}+\kappa_{2}\right)
$$

which comes from theorem 5(iv) in Madras and Whittington (2003), and uses recent numerical estimates of $\kappa_{3}$ (MacDonald et al 2000) and $\kappa_{2}$ (Jensen and Guttmann 1999). The bound $\beta=\alpha$ comes from corollary 3 of Madras and Whittington (2003). The curve in the first quadrant for $\alpha \geqslant \gamma-\log \left(2-\mathrm{e}^{\gamma}\right)$ is

$$
\beta=\log \left(2-\mathrm{e}^{-\alpha}\right)
$$

which is a lower bound on $\beta_{c}(\alpha, \gamma)$, for $\gamma<\log 2$, obtained by the partial annealing argument used in theorem 8 of Madras and Whittington (2003). The vertical dashed line in the first and 


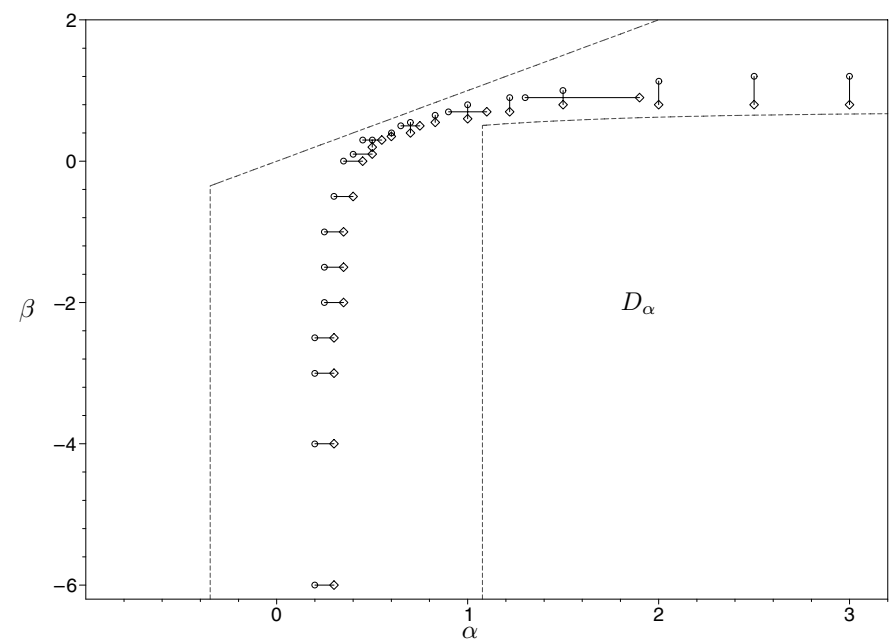

Figure 2. The phase diagram in the $(\alpha, \beta)$-plane for $\gamma=0.4$. The symbols, lines and bounds are otherwise as in figure 1 .

fourth quadrants is $\alpha=\gamma-\log \left(2-\mathrm{e}^{\gamma}\right)$ and is a bound on the phase boundary obtained from corollary 9(ii) of Madras and Whittington (2003). Note that the phase boundary does not pass through the origin and hence the phase boundaries of $D_{\alpha}$ and $D_{\beta}$ are disjoint. This implies that $\gamma_{1}<0.2$. Since part of the phase boundary is in the third quadrant, the results suggest that $\gamma_{2}>0.2$. We shall return to estimating the values of $\gamma_{1}$ and $\gamma_{2}$ in the next section. In figure 2 we show the corresponding phase boundary for $\gamma=0.4$. The phase boundary has shifted to the right and now is entirely outside the third quadrant (we checked this for values of $\beta$ down to -12 ) so $\gamma_{2}$ appears to be less than 0.4 . We again show the bounds derived from the results of Martin et al (2000) and Madras and Whittington (2003). The lower bound on the location of the phase boundary in the first quadrant is quite effective at values of $\alpha$ larger than about 1.1 .

\section{Estimates of the critical values of $\gamma$}

We recall that there exists a value $\gamma_{1}$ such that the point $(0,0, \gamma)$ is in the interior of the localized phase for all $\gamma>\gamma_{1}$ and that it is known that $\gamma_{1} \leqslant 2 \kappa_{3}-\kappa_{2}-\sinh ^{-1} \cosh \kappa_{3}$ which is approximately 0.49 , using recent numerical estimates of $\kappa_{3}$ (MacDonald et al 2000) and $\kappa_{2}$ (Jensen and Guttmann 1999). One would expect that $\gamma_{1}=0$ (De'Bell and Lookman 1993) but there is no proof of this, and the bound given above is the best that is known rigorously. In order to estimate $\gamma_{1}$ numerically we have made use of that fact that $\bar{\kappa}(0,0,0)=\kappa_{3}$ and $\bar{\kappa}(0,0, \gamma)>\kappa_{3}$ for every $\gamma>\gamma_{1}$. We have used ratio analysis methods to estimate $\bar{\kappa}(0,0, \gamma)$ for values of $\gamma$ between zero and 0.49 in an attempt to find smaller values of $\gamma$ where we can be reasonably certain that the value of $\bar{\kappa}(0,0, \gamma)$ is greater than $\kappa_{3}$. In figure 3 we show the results of a ratio analysis for $\alpha=\beta=0, \gamma=0.075$ where we plot $R_{n}$ as well as $Y_{n}(\theta)$ for $\theta=43 / 32$ and for $\theta=0.68$. We also show linear extrapolants of $R_{n}$, i.e. $L_{n}=\left(n R_{n}-(n-2) R_{n-2}\right) / 2$. (Note that equation (2.2) implies that $L_{n}=\mathrm{e}^{\bar{\kappa}}(1+o(1))$.) There is strong evidence that these four plots (which must have a common intercept) converge to a value larger than $\kappa_{3}$ indicating that the point $(0,0, \gamma)$ is in the localized phase when $\gamma=0.075$, and hence that $\gamma_{1}<0.075$. In figure 4 , estimates of $\mathrm{e}^{\bar{\kappa}(0,0, \gamma)}$ based on extrapolation of the ratios are presented for $\gamma \geqslant 0$; 


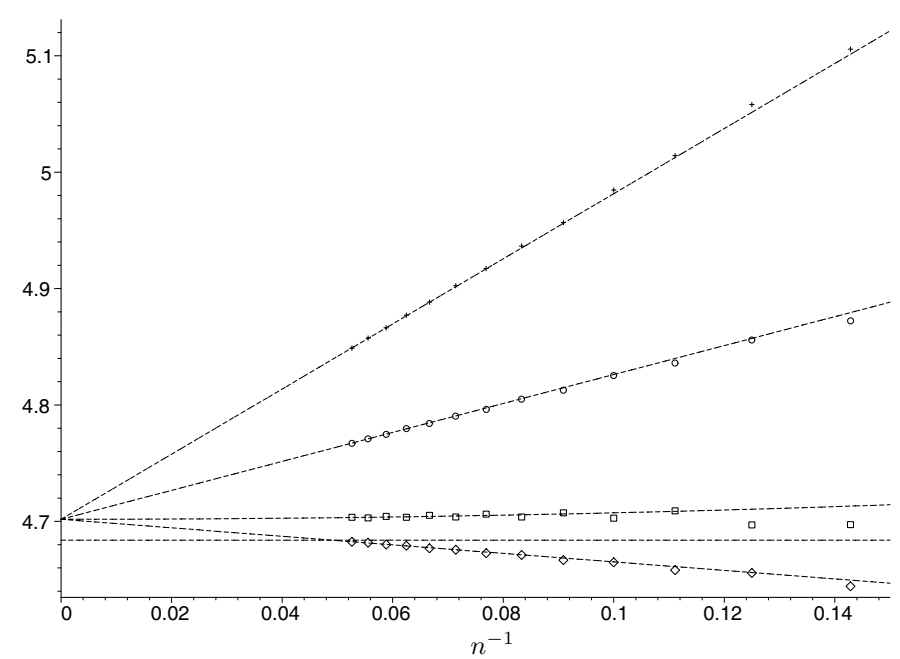

Figure 3. The ratios $Y_{n}(0.68)(+), R_{n}=Y_{n}(1)(\circ), Y_{n}(43 / 32)(\diamond)$ and the linear extrapolant, $L_{n}(\square)$, are shown for $\alpha=0, \beta=0, \gamma=0.075$. The lowest horizontal dashed line is the line $y=\mathrm{e}^{\kappa_{3}}$ and the other dashed lines are sample linear fits to the ratios and a sample quadratic fit to the linear extrapolant.

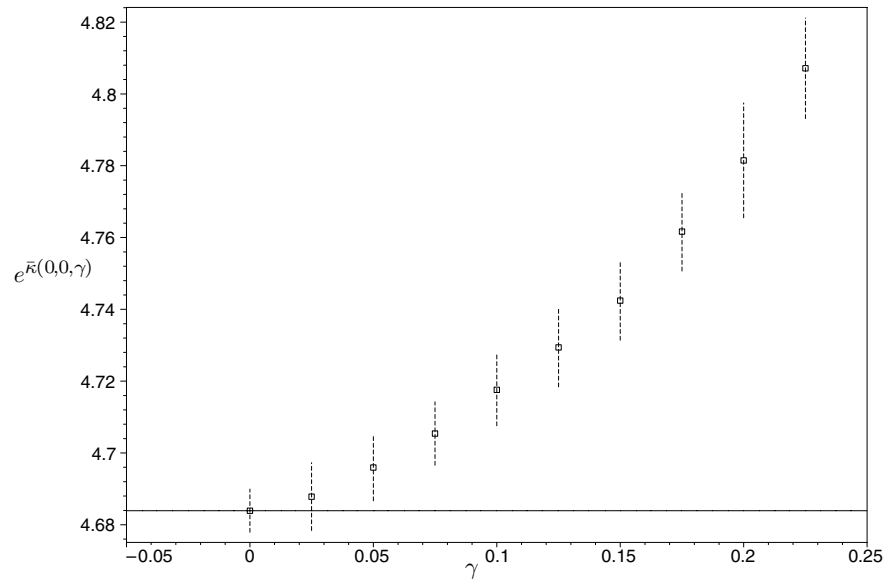

Figure 4. The free energy estimates for $\alpha=0, \beta=0$.

these results are consistent with the conjecture that $\gamma_{1}=0$. Our error bars are based on several types of fit which include $Y_{n}(43 / 32)$ having zero limiting slope.

If $\gamma>\gamma_{2}$ the third quadrant of the $(\alpha, \beta)$-plane is entirely in the localized phase (so that the phase boundary does not enter this quadrant). We know that $\gamma_{2} \leqslant \kappa_{3}-\kappa_{2} \approx 0.574$, using numerical estimates of $\kappa_{3}$ and $\kappa_{2}$. Figures 1 and 2 give numerical evidence that $0.2<\gamma_{2}<0.4$, but we have tried to obtain a more precise value of $\gamma_{2}$ by estimating the value of $\bar{\kappa}(0, \beta, \gamma)$ for large negative values of $\beta$ and for values of $\gamma$ between 0.2 and 0.4 , and comparing to $\kappa_{3}+\alpha / 2$, the value when delocalized into the $\alpha$-phase. Figures 1 and 2 suggest that taking a value of $\beta=-6$ is probably sufficient. To complement this approach we have also investigated $\beta=-\infty$. To justify this, James et al (2003) proved that for the quenched average free energy, 
$\left\langle\kappa_{n}(\alpha, \beta, \gamma \mid \chi)\right\rangle$, the order of the two limits $\beta \rightarrow-\infty$ and $n \rightarrow \infty$ can be interchanged. Hence we can first set $\beta=-\infty$ and then extrapolate to large $n$ to investigate the value of

$$
\bar{\kappa}(\alpha,-\infty, \gamma) \equiv \lim _{\beta \rightarrow-\infty} \lim _{n \rightarrow \infty}\left\langle\kappa_{n}(\alpha, \beta, \gamma \mid \chi)\right\rangle .
$$

We next explain how this approach can be used to obtain estimates for both $\gamma_{2}$ and $\gamma_{3}$.

Define

$$
\gamma_{c}(\alpha, \beta) \equiv \sup \left\{\gamma:(\alpha, \beta, \gamma) \in D_{\alpha}\right\}
$$

for any fixed $\alpha \leqslant \infty$ and $\beta \geqslant-\infty$ such that the set of $\gamma$ on the right-hand side is not empty. We note that for $\beta_{1}>\beta_{2}$, if $\left(\alpha, \beta_{1}, \gamma\right) \in D_{\alpha}$ then $\left(\alpha, \beta_{2}, \gamma\right) \in D_{\alpha}$. Note also from theorem 2 of Madras and Whittington (2003) that $\left(\alpha, \beta, \gamma_{c}(\alpha, \beta)\right) \in D_{\alpha}$. Hence for $\beta_{1}>\beta_{2}$ we have that $\left(\alpha, \beta_{1}, \gamma_{c}\left(\alpha, \beta_{1}\right)\right) \in D_{\alpha}$ and hence $\left(\alpha, \beta_{2}, \gamma_{c}\left(\alpha, \beta_{1}\right)\right) \in D_{\alpha}$. Then, the definition of $\gamma_{c}(\alpha, \beta)$ implies that $\gamma_{c}\left(\alpha, \beta_{2}\right) \geqslant \gamma_{c}\left(\alpha, \beta_{1}\right)$. Thus

$$
\sup _{\beta>-\infty} \gamma_{c}(\alpha, \beta)=\lim _{\beta \rightarrow-\infty} \gamma_{c}(\alpha, \beta) \leqslant \gamma_{c}(\alpha,-\infty)<\infty
$$

where the last inequality follows from theorem 11 of Madras and Whittington (2003). We expect and assume that

$$
\lim _{\beta \rightarrow-\infty} \gamma_{c}(\alpha, \beta)=\gamma_{c}(\alpha,-\infty) .
$$

A similar argument, using the fact that $\left(\alpha_{2}, \beta, \gamma\right) \in D_{\alpha}$ implies $\left(\alpha_{1}, \beta, \gamma\right) \in D_{\alpha}$ for $\alpha_{1}>\alpha_{2}$, can be used to show that

$$
\sup _{\alpha<\infty} \gamma_{c}(\alpha, \beta)=\lim _{\alpha \rightarrow \infty} \gamma_{c}(\alpha, \beta) \leqslant \gamma_{c}(\infty, \beta)<\infty
$$

where the last inequality follows from theorem 11 of Madras and Whittington (2003). We expect and assume that

$$
\lim _{\alpha \rightarrow \infty} \gamma_{c}(\alpha, \beta)=\gamma_{c}(\infty, \beta) .
$$

Note that

$$
\gamma_{2} \equiv \sup \left\{\gamma:(\alpha, \beta, \gamma) \in D_{\alpha} \text { for some } \alpha \leqslant 0,-\infty<\beta \leqslant \alpha\right\}
$$

and

$$
\gamma_{3} \equiv \sup \left\{\gamma:(\alpha, \beta, \gamma) \in D_{\alpha} \text { for some } \alpha<\infty,-\infty<\beta \leqslant \alpha\right\} .
$$

Then from equation (3.2) we have

$$
\gamma_{2}=\sup \left\{\gamma_{c}(\alpha, \beta): \alpha \leqslant 0,-\infty<\beta \leqslant \alpha\right\}
$$

and

$$
\gamma_{3}=\sup \left\{\gamma_{c}(\alpha, \beta): \alpha<\infty,-\infty<\beta \leqslant \alpha\right\} .
$$

However since $\gamma_{c}(\alpha, \beta)$ is non-increasing in $\beta$ and non-decreasing in $\alpha$, therefore

$$
\gamma_{2}=\lim _{\beta \rightarrow-\infty} \gamma_{c}(0, \beta)
$$

and

$$
\gamma_{3}=\lim _{\alpha \rightarrow \infty} \lim _{\beta \rightarrow-\infty} \gamma_{c}(\alpha, \beta)
$$

where for the first equation the fact that $\gamma_{c}(\alpha, \beta)$ is continuous at $\alpha=0$ has been used (this follows from theorem 2 of Madras and Whittington (2003)). This is the route that we have followed in estimating $\gamma_{2}$. In fact it is convenient to determine the approximate location of the transition curve in the $(\alpha, \gamma)$-plane when $\beta=-\infty$ since this yields estimates both of $\gamma_{2}$ 


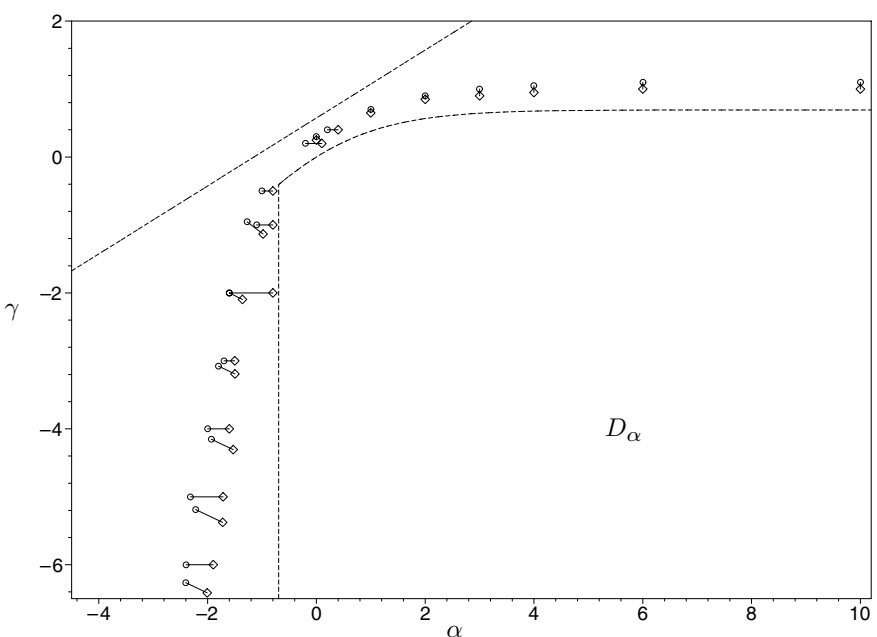

Figure 5. The phase diagram in the $(\alpha, \gamma)$-plane, with $\beta=-\infty$. The phase boundary, $\gamma_{c}(\alpha, \beta)$, is expected to cross each of the solid lines and the symbols $\diamond$ and $\circ$ have the same meaning as in figure 1 .

and $\gamma_{3}$. In particular, in the $(\alpha, \gamma)$-plane when $\beta=-\infty$, assumptions (3.4) and (3.6) and equation (3.12) imply that the horizontal asymptote is $\gamma=\gamma_{3}$ and that $\gamma_{3}=\gamma_{c}(\infty,-\infty)$.

For $\beta=-\infty$ and a given colouring $\chi$, the only walks which have a positive probability of occurring are those which have no $B$ monomers in the $\beta$-phase (i.e. $z<0$ ). Hence we define the partition function

$$
Z_{n}(\alpha,-\infty, \gamma \mid \chi)=\sum_{v_{A}, w} c_{n}\left(v_{A}, 0, w \mid \chi\right) \mathrm{e}^{\alpha v_{A}+\gamma w}
$$

and the corresponding free energy

$$
\kappa_{n}(\alpha,-\infty, \gamma \mid \chi)=n^{-1} \log Z_{n}(\alpha,-\infty, \gamma \mid \chi) .
$$

Note that for $\beta=-\infty, \max \{\alpha / 2, \beta / 2\}=\alpha / 2$ for all finite $\alpha$ so that the region $D_{\beta}$ does not exist except possibly at $\alpha=-\infty$. Our estimate of the location of the phase boundary in the $(\alpha, \gamma)$-plane at $\beta=-\infty$ is given in figure 5. The upper bound on the phase boundary (a dashed line with positive slope) is

$$
\gamma=\kappa_{3}-\kappa_{2}+\alpha / 2
$$

using numerical estimates for $\kappa_{3}$ and $\kappa_{2}$, and follows from theorem 5(iii) of Madras and Whittington (2003). The lower bound (a dashed curve and vertical line) is

$$
\begin{array}{ll}
\gamma=\log \left(\frac{2}{1+e^{-\alpha}}\right) & \text { for } \alpha \geqslant-\log 2 \\
\alpha=-\log 2 & \text { for } \gamma \leqslant \log (2 / 3)
\end{array}
$$

which follows from the proof of theorem 8 of Madras and Whittington (2003). Define

$$
\alpha_{c}(\beta, \gamma) \equiv \inf \left\{\alpha:(\alpha, \beta, \gamma) \in D_{\alpha}\right\}
$$

for any fixed $\beta \geqslant-\infty$ and fixed $\gamma \geqslant-\infty$ such that the set of $\alpha$ on the right-hand side is not empty. Note that arguments analogous to those which led to equation (3.3) can be used to show that $\alpha_{c}(\beta, \gamma)$ is a non-decreasing function of both $\beta$ and $\gamma$. We note further that 
for fixed finite $\beta$, theorem 6 of Madras and Whittington (2003) implies that there is a vertical asymptote, $\alpha=\alpha_{A}^{*}(\beta) \equiv \lim _{\gamma \rightarrow-\infty} \alpha_{c}(\beta, \gamma)=\inf _{\gamma>-\infty} \alpha_{c}(\beta, \gamma) \geqslant \beta$, in the $(\alpha, \gamma)$-plane. James et al (2003) have proved that for $-\infty \leqslant \beta \leqslant \alpha<\infty$

$\bar{\kappa}(\alpha, \beta,-\infty) \equiv \lim _{\gamma \rightarrow-\infty} \lim _{n \rightarrow \infty}\left\langle\kappa_{n}(\alpha, \beta, \gamma \mid \chi)\right\rangle=\lim _{n \rightarrow \infty} \lim _{\gamma \rightarrow-\infty}\left\langle\kappa_{n}(\alpha, \beta, \gamma \mid \chi)\right\rangle=\kappa_{3}+\alpha / 2$.

Hence we expect (assuming the continuity of the phase boundary at $\gamma=-\infty$ ) that $\alpha_{A}^{*}(\beta)=\beta$ for finite $\beta$ and that there is no vertical asymptote for $\beta=-\infty$. Figure 5 is consistent with this.

For our estimate of $\gamma_{2}$, it was found that the point $(0,-\infty, 0.25) \in D_{\alpha}$ while for the point $(0,-\infty, 0.3)$ the system was localized. Hence we estimate $0.25 \leqslant \gamma_{2} \leqslant 0.3$.

To estimate $\gamma_{3}$ we have estimated the horizontal asymptote in figure 5 . This was based on looking at the free energy estimates for $\alpha$ large, e.g. $\alpha=20$, and also by setting $\alpha=\infty$. In the latter case, we use the results of James et al (2003) which prove that for any $\beta<\infty$ the order of the limits $n \rightarrow \infty$ and $\alpha \rightarrow \infty$ can be interchanged for the modified quenched average free energy $\bar{\kappa}_{n}^{\prime}(\alpha, \beta, \gamma)=\left\langle\kappa_{n}(\alpha, \beta, \gamma \mid \chi)\right\rangle-\alpha / 2$. For the case $\beta=-\infty$ and $\alpha=\infty$, the only walks which contribute to $\bar{\kappa}_{n}^{\prime}(\infty,-\infty, \gamma)$ are those in $z \geqslant 0$ in which all the $A$-vertices are in $z>0$. From an analysis of extrapolants of $\bar{\kappa}_{n}^{\prime}(\infty,-\infty, \gamma)$ (compared with $\kappa_{3}$ ), we conclude that $1 \leqslant \gamma_{3} \leqslant 1.1$.

\section{Discussion}

We have used exact enumeration and series analysis methods to estimate the locations of the phase boundaries for a self-avoiding walk model of a random copolymer localizing at the interface between two liquid phases. Our results give quantitative estimates which confirm the qualitative features predicted by Martin et al (2000) and Madras and Whittington (2003). We have incorporated the effect of an interaction $(\gamma)$ of the vertices of the walk with the interfacial plane between the two liquid phases and have estimated the critical values of $\gamma$ where (i) the point $(0,0, \gamma)$ becomes localized, (ii) all of the third quadrant of the $(\alpha, \beta)$-plane becomes localized and (iii) the whole $(\alpha, \beta)$-plane becomes localized.

Many questions remain open. Settling the question as to whether or not $\gamma_{1}$ is zero is closely related to the open question of the location of the adsorption transition for the selfavoiding walk model of homopolymer adsorption at a penetrable surface (Hammersley et al 1982, De'Bell and Lookman 1993).

We have said nothing about the order of the localization transition. Causo and Whittington (2003) studied this question by Monte Carlo methods and found evidence that, when $\gamma$ is zero, the order of the transition is different in the first and third quadrants. Presumably the origin in the $(\alpha, \beta)$-plane is a multi-critical point where the order changes, though this is not known. What happens when $\gamma>\gamma_{1}$, so that the phase boundaries do not have a common point at the origin? This problem could be addressed by Monte Carlo methods.

\section{Acknowledgments}

This research was supported, in part, by NSERC of Canada. Some of this work was carried out while CES was visiting the Department of Chemistry at the University of Toronto and she is grateful to the members of that department for their hospitality. 


\section{References}

Biskup M and den Hollander F 1999 Ann. Appl. Probab. 9 668-87

Bolthausen E and den Hollander F 1997 Ann. Probab. 25 1334-66

Brout R 1959 Phys. Rev. 115 824-35

Causo M S and Whittington S G 2003 J. Phys. A: Math. Gen. 36 L189-L195

De'Bell K and Lookman T 1993 Rev. Mod. Phys. 65 87-113

Gaunt D S and Guttmann A J 1974 Phase Transitions and Critical Phenomena vol 3, ed C Domb and M S Green (London: Academic) pp 181-243

Hammersley J M 1957 Proc. Camb. Phil. Soc. 53 642-5

Hammersley J M, Torrie G M and Whittington S G 1982 J. Phys. A: Math. Gen. 15 539-71

James E W, Soteros C E and Whittington S G 2003 J. Phys. A: Math. Gen. accepted

Jensen I and Guttmann A J 1999 J. Phys. A: Math. Gen. 32 4867-76

MacDonald D, Joseph S, Hunter D L, Moseley L L, Jan N and Guttmann A J 2000 J. Phys. A: Math. Gen. 33 $5973-83$

Madras N and Whittington S G 2003 J. Phys. A: Math. Gen. 36 923-38

Maritan A, Riva M P and Trovato A 1999 J. Phys. A: Math. Gen. 32 L275-280

Martin R, Causo M S and Whittington S G 2000 J. Phys. A: Math. Gen. 33 7903-18

Sinai Ya G and Spohn H 1996 Am. Math. Soc. Transl. Ser. 2177 219-23

Vanderzande C 1998 Lattice Models of Polymers (Cambridge: Cambridge University Press) 